\title{
HL7-SAIF in motion A pragmatic perspective
}

\author{
Sabar MI, Jayaweera PM \& Edirisuriya EATA
}

Department of Computer Science, University of Sri Jayewardenepura, Gangodawila, Sri Lanka

\begin{abstract}
Health Level 7 (HL7) is the most popular global health care standard in operation today. It provides an Enterprise Architecture (EA) for the exchange, integration, sharing, and retrieval of electronic health information. Closely allied is the Services-Aware Interoperability Framework (SAIF) which is the Interoperability Framework that operates on HL7. Using the Messaging, Document Exchange, and Services paradigms, SAIF represents the careful blend of the best practices and concepts of many architectural frameworks. Utilizing SAIF to churn out HL7-based EA specifications, ensure inter-enterprise and intraenterprise, component-wise, cross-referenced, consistency, conformity, and compliance. This is true irrespective of the interoperability paradigm used ,ie., Messages, Documents, or Services. However, these technologies are not without their problems, and cynics. They have documented design and implementation issues, both empirical and practical. The thrust of this paper is to present the "case technology" of HL7SAIF, both conceptual and engineered, highlighting the shortcomings, design issues, and practical difficulties encountered during specifications design and development. Further, pertinent solutions devised in this research to overcome these pressing issues are also articulated.
\end{abstract}

KEYWORDS: Enterprise Architecture, Semantic Interoperability, HER

Corresponding author: M. I. Sabar, Email: ishan.res@gmail.com 


\section{INTRODUCTION}

Presently, many Enterprise Architecture Frameworks (EAFs) are in use globally, eg., Reference Model for Open Distributed Processing (RM-ODP), The Open Group Architecture Framework (TOGAF), and Zachman. However, these three EAFs do not place emphasis upon Working Interoperability (WI), the ability for different enterprise systems to exchange and use pertinent information in a systematic, well-defined, meaningful way. This applies to both inter-enterprise (amongst Enterprise systems) and intra-enterprise (within components of a particular Enterprise system) interoperability.

Conversely, SAIF was especially devised with WI in mind, promoting the development of Enterprise Architecture Specifications (EAS) for mutative, dynamic EA-modelled environments. HL7 is one such EA, a standard which serves the healthcare sector. According to (HL7, 2010), HL7 provides a framework for the exchange, integration, sharing, and retrieval of electronic health information. SAIF on the other hand is a meaningful blend of many popular EA-related methodologies such as ServicesOriented Architecture (SOA), Object Management Group's (OMG) Model-Driven Architecture (MDA), Computable Semantic Interoperability (CSI), and the ISO's Reference Model for Open Distributed Processing (RMODP). Indeed, it represents a delicate, but immensely potent blend of these technologies.

Noteworthy is the fact that EA-related HL7 is an "international healthcare informatics interoperability standard"(HL7, 2012) whilst SAIF operates on generic, multi-disciplinary EA realms which elicit Semantic Working Interoperability. In fact the HL7-SAIF technology blend represents the epitome' of smart "customization"; SAIF adapted to operate in the healthcare provision sector rendering specialized enterprise-related, HL7-driven "informatics interoperability" (HL7, 2012).

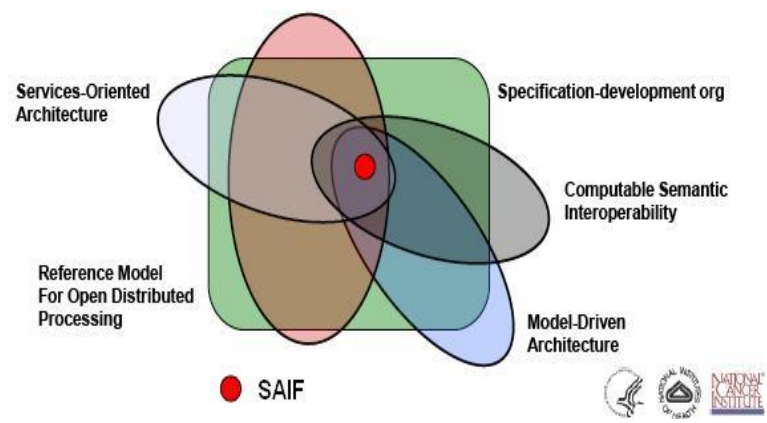

Figure 1. Lens of SAIF (NCI, 2012)

Unfortunately, many design and development issues have arisen, or have been detected in relation to HL7-SAIF. These issues require a unified, multi-aspect approach to remediation. The objective of this paper is to firstly present the salient and significant concepts and attributes of HL7-SAIF technology, highlighting is merits and demerits in specifications generation. The reader is given an inkling of the numerous design and implementation issues surrounding HL7-SAIF. For instance, the current HL7 version v3 has been found to be complex and difficult to implement and operate. V3 which is XMLbased, has been promoting Semantic Interoperability defined as the ability of two or more computer systems to communicate information with homogenous understanding. Central to this goal is HL7's Reference Information Model (RIM) which has been found to be "lacking and deviant from the accepted norms of modelling" (Landgrebe et al., 2011).

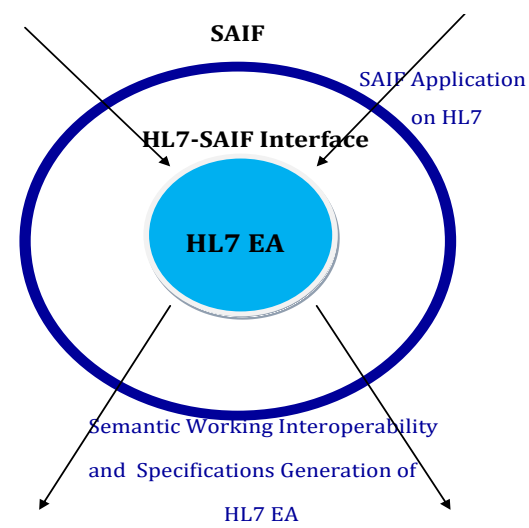

Figure 2. Layered HL7-SAIF Operation 
Thereafter, the multi-aspect remediation approaches devised in our present research, are presented. These solutions are pertinent to the issues touched on above, particularly with respect to HL7.Undoubtedly, the enhancements accrued by the mentioned 'fixes' would permeate and overarch, rendering HL-SAIF as a ubiquitous, viable, and cost-effective solution in any environment.

Thus, this paper is organized as follows; Section 2 of this paper focuses on HL7 and its inherent characteristics. Section 3 describes the HL7SAIF methodology in operation. Section 4 highlights the many structural and operational issues currently plaguing HL7-SAIF technology. Section 5 articulates flavours of the multi-aspect solutions devised in respect of the preceding issues. In conclusion, section 6 reviews and put forth further recommendations to make HL7-SAIF technology more robust and secure.

\section{BACKGROUND}

"Health Level 7 (HL7) is a collection of standards and proposals for healthcare-specific data exchange between computer applications" (HL7, 2014). It lays down strict modalities for the exchange, integration, sharing, and retrieval of electronic health information (HL7 2013). Level Seven refers to the relevant layer of the ISO's (International Organization for Standardization) seven-layer communications model for Open Systems Interconnection (OSI), the Application Level"(HL7, 2012).

Healthcare service providers worldwide use a veritable plethora of computer-based information exchange systems; their applications and functionalities being eclectic, running on varied hardware and software platforms. The difficulty has been to streamline and afford the free flow and exchange of medical information such as patient, disease, medication, and laboratory test records between these far-flung computer systems, in an expeditious, efficacious, consistent, and precise manner. "HL7 specifies a number of flexible standards, guidelines, and methodologies by which various healthcare systems can communicate with each other" (HL7, 2010). These standards prescribe guidelines for the sharing, exchange, and use of healthcare-related information amongst the various participating healthcare enterprises.

According to (HL7, 2012), HL7 standards are multi-functional :

1. Conceptual, Data-related standards eg., HL7 RIM.

2. Document standards eg., HL7 CDA meaning Clinical Document Architecture.

3. Application standards eg., HL7 CCOW meaning Clinical Context Object Workgroup.

4. Messaging Standards eg., HL7 v2.x and v3.0.

(HL7, 2012) further elaborates on the above as follows :

"HL7 Messaging standards are particularly important because they define how information is packaged and communicated from one party to another. These standards set the language, structure, and data types required for seamless integration from one system to another. The Reference Information Model (RIM) and the HL7 Development Framework (HDF) are the basis of the HL7 v3 standards development process".

In particular, the character-delimited version 2.x of HL7 remediated many existing issues in the healthcare service Information Technology (IT) realm, back in the 1990's. It supplanted many unorganized, impromptu information exchange protocols and standards prevalent at the time. However, its value was limited by the fact that it dealt with Syntactic Interoperability, which was "merely a syntactic exchange format allowing applications to parse contents of an electronic message reliably" (Landgrebe etal., 
2011). Syntactic Interoperability "guarantees the exchange of the structure of the data but carries no assurance that all parties will interpret the meaning identically" (HL7, 2010). This is a conventional point-to-point connection and Distributed Computing is not supported. However, despite its "no frills" functionality, it proved to be a stellar performer in the healthcare enterprise interoperability circuit which a large client base. It is still the predominant information exchange related standard in the healthcare service sector today.

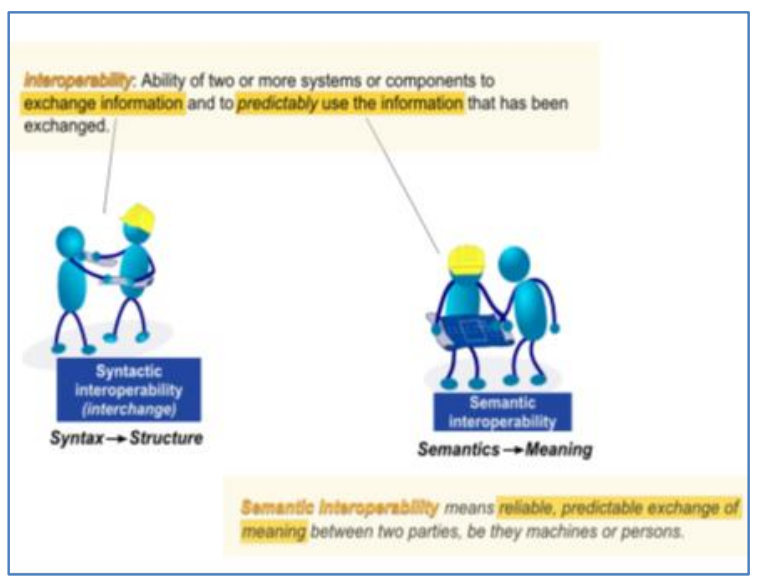

Figure 3. Syntactic versus Semantic Interoperability (HL7, 2010)

Version 3 (v3) of HL7 enhanced this approach to cover Semantic Working Interoperability (SWI) in the EA. The Reference Information Model (RIM) was developed by HL7 in order to facilitate this effort. The proponents exalted this new thinking, but opponents were less enthusiastic forwarding the notion that v3 efforts would fall short of expected benefits, if not fail completely.

The forwarded reasoning as spelled out in (HL7, 2014) is given below:

1. It is not possible to devise a universal, optimal data model that would cater to every EA's needs. The model is a function of the particular EA and its operating environment; thus the single all-acceptable model approach would fail.
2. The backbone of the data modeling effort in v3 is the Reference Information Model (RIM). This methodology has been at the receiving end of much criticism; its semantic inconsistency and its deviation from accepted data modeling methodology being the main "nay" argument.

3. V3 is complex and impractical to implement and maintain.

Hence, opponents argue that only the wealthier economies and affluent multinational business corporations can afford a implementation of v3. Indeed, this has been the trend in the healthcare sector worldwide. Many enterprises have been slow in migrating to HL7 v3; most clients have been happy to "live" with the antiquated but tried-and-tested v2, trusted for its correctness and behaviour despite its more limited functionality, versatility, and scope. It has been established that version 2.x "which supports clinical practice and the management, delivery, and evaluation of health services, is the most commonly used in the world" (HL7, 2010)..

Presently, HL7 (in particular v3) has assumed global significance and stature as a renowned healthcare-related, interoperability-driven information exchange standard. It has been further established that HL7's RIM-based attempt at achieving SWI was unsuccessful, and this lead to the development of SAIF to achieve this end.

\section{HL7-SAIF ISSUES}

Many problems exist with the current design and implementations of HL7. These affect the quality, precision, and pertinence of the HL7SAIF specifications derived in a given EA environment, which in turn snowball to subvert global Semantic Interoperability and inclusive efficiency, our principle goals. This research analyzed the HL-SAIF specifications development sub-processes in great depth, and devised appropriate remediation methods to 
confront and solve these pressing issues; indeed the primary focus was to formulate a unified and sound solution to the many, multi-aspect HL-SAIF related development and implementation issues.

i. According to (Landgrebe et al., 2013), HL7 does not afford the ability to formalise requirements of business processes. "This means that SAIF-conformant information models may be created without a traceable link to formalised requirements, which would preclude the achievement of interoperability".

ii. The HL7 specifications development subprocesses require analysis and enhancement. In particular, this study determined that any upper ontology enhancement would ripple down to subsequent HL7 specifications development sub-processes promoting overarching and inclusive efficiency in the specifications generation continuum.

iii. HL7 v3 message structures represented in XML (Extended Markup Language) were found to be wordy and syntactically superfluous at times. A compact, structured message format would significantly enhance line efficiency and throughput.

iv. Current Electronic Health Record (EHR) schemes although vastly improving performance in comparison to conventional database-driven healthcare networks, still lacked panache to capitalize on the immense potential shown at inception; many possibilities are left unutilized or underutilized.

v. Finally, the most significant issue concerns the affordability and remedial potential of HL7 in the local Sri Lankan healthcare sector, and indeed its extrapolation to the greater Asian and even African contexts. Why and how should we implement the HL7 standard in our national health services and related healthcare systems? Is this exercise incentive enough for the poorer economies of the world?
Listed above were a few of the numerous issues and concerns that have been detected with regard to HL7-SAIF technology. Thus, a significant research effort is needed to filter out the flaws and inject remediation and enhancement, to generate a coherent, consistent, precise, HL7-SAIF based, global electronic healthcare information exchange service.

\section{RESEARCH METHODOLOGY AND MATERIALS}

"The Health Level 7 Service-Aware Interoperability Framework (HL7-SAIF) provides consistency between all artifacts and enables a standardized approach to EA development and implementation", and affords a way to measure this consistency (HL7, 2013). The EA referred to in this case is HL7. HL7SAIF "seeks to meet this need by providing a framework ensuring interoperability for documents, messages, and services between organizations" in the healthcare service sector (HL7, 2010).

It is pertinent that HL7 refers to standards related to electronic information exchange and their subsequent meaningful use (interenterprise and/or intra-enterprise) in the healthcare service sector. On the other hand SAIF per se is an multi-disciplinary enterprise architecture framework (EAF) for generation of semantic working interoperability (SWI) related specifications, ie., it can be applied to any generic EA exhibiting inter-enterprise and/or intra-enterprise level SWI. HL7-SAIF refers to the particular application of SAIF to the healthcare service sector EA HL7.

The research methodology used herein therefore involved the complete and exhaustive use of the Design Science (DS) research verification and validation paradigm. Since the principle goal of this research was to devise a unified, inclusively-efficient solution to the HL7-SAIF specifications development paradigm, each "fix" or enhancement was subjected to stringent 
verification and validation testing, prior to Incremental Integration. The application of the DS validation paradigm was at two levels, ie., at each correction or enhancement level, and at the final integration level.

This paper therefore presents and reviews the results of several studies conducted successfully by our team, the findings of which have been already published in several eminent fora.

\section{RESULTS}

The research conducted focused primarily on the principle aspects of HL7-oriented healthcare systems development and applicability. Besides focusing on the underlying framework of the standard, its representation, internals of the specifications development continuum, and specifics of inter-enterprise communication, this study's thrust also explored and successfully devised globally-consolidated EHR schemes, thereby promoting HL7's usefulness and applicability to the local, and greater Asian context.

Requirements Traceability to antecedent domain requirements from finalized specifications, was actualized by representing all UML-based (Unified Modelling Language) DAM (Domain Analysis Model) artifacts in the newly-devised, proposed Unified Data Atom(UDA) representation, either first-hand or as a single-step transliteration (Sabar et al., 2015-1). It was proved in the study that the UML-based DAM to UDA transformation is trivial. Data Element, Classes and Attributes, State Machines, Storyboards, Activities, Interactions, and Use Cases constitute seven DAM structures, which are transformed to the analogous UDA representation, ensuring overarching uniformity and homogeneity in the modelled domain specifics. Indeed, this induced uniformity overarches laterally across all DAM structures and longitudinally across all phases, as the DAM heads towards terminal specifications. The UDA schema derivation process is described below. The Necessary condition for transformation $\mathrm{T}^{\mathrm{w}}$ is stated as follows (Sabar et al., 2015-1) :

If UDA signifies the set of transliterated, target DataAtoms $\left\{\mathrm{u}_{1}, \mathrm{u}_{2}, \mathrm{u}_{3}, \mathrm{u}_{4}, \ldots \ldots \ldots \ldots \ldots, \mathrm{u}_{\mathrm{k}}\right\}$ as a result of the Complete Transformation T ${ }^{\omega}$ acting on the source UML informational schema $U$ where $\left\{d_{1}, d_{2}, d_{3}, \ldots \ldots \ldots, d_{1}\right\} \in U$ represent source DAM artifact elements, then

$$
\begin{aligned}
T^{w}: U & \rightarrow U D A \quad U \subseteq \mathrm{U} \text { and } U D A \subseteq \mathrm{U}^{\prime} \\
& \text { where } \\
& \mathrm{U} \text { - Problem domain } U M L \text { super } \\
& \text { schema, and } \\
& \mathrm{U} \text { - Problem-related target } U D A \text { super } \\
& \text { schema }
\end{aligned}
$$

It was proved that the mapping

$$
\mathrm{T}^{\mathrm{w}}: \mathrm{U} \rightarrow \mathrm{UDA}
$$

denotes a Complete Transformation. The proposed solution also utilizes strategicallyplaced, state-related Annotation-Posts (APs) to capture and record in-place every state mutation, eg., a RIM-related annotation. These APs act as Scoreboards to the specifications generation continuum, affording precision coordinate instantiation for onward and backward navigation between the domain and finalized specifications.

Upper Ontology analysis determined that stakeholder analysis and design interoperability can be achieved by inducing representational homogeneity in the HL7 framework and allied phases within ripple-distance (Sabar et al., 2015-2). This research focused on the belief that the achievement of true global interoperability is rooted at the labyrinths of specifications development; Infusing simplicity and uniformity in communications would derive nascent analysis, design, and semantic interoperability, which in turn would permeate to true International Interoperability and inclusive efficiency in system operation. Multi- 
aspect interoperability injection in core processes promote and enhance numerous allied activities, from domain requirements crosschecking, audit, and consensus, to kindred system development verification and validation. Hence, the proposed UDA vocabulary and its interpolation is a significant first step towards achieving true techno-platform independence in HL7 ontology representations.

The Green Messages solution was devised for purely "in the wire" efficiency in the overall healthcare network of participating enterprises (Sabar et al., 2015-3). HL7 v3 message structures represented in XML (Extended Markup Language) were found to be wordy and syntactically superfluous at times. A compact, structured message format would significantly enhance line efficiency and throughput. Indeed, this was what was achieved using the proposed Green Messages approach. Verbose v3 message structure representations were meaningfully abridged, whilst strictly maintaining their original nuance and flavour. The Greening Axiom devised and shown below, articulates the process.

\section{Greening Axiom}

"The greened target message contains 'only the most pertinent' v3 source message elements needed for qualitative content communication, ie., those the specified interaction 'cannot do without'. All other superfluous and ancillary source message elements are disregarded in the greening Transformation". "Each semantically identifiable source XML Message element is processed as above and corresponding target determined. The resulting, abridged, XMLbased target Message stream constitutes the Green Message”.

The derived target Green Message structures have to be overarching and truly universal, affording and facilitating International Interoperability. Indeed, their brevity ensures compactness and higher packing density, lending seamlessly to greater line throughput.
Aligned to the v3 message Greening process are more efficient procedures for secured, efficacious exchange of data and information "in the wire" enhancing overall efficiency and promoting true International Interoperability in operation.

Electronic Health Records (EHRs) are electronically maintained, contiguous records of patient-related healthcare information collected during one or many patient encounters (Sabar et al., 2015-4). The EHR facilitates the precision diagnosis, treatment, and decision support processes of patient care. Since EHR design and implementation is still evolving, many aspects are under-used or under-utilized. Its implementation is primarily confined to national pockets, managed by individual National Health Systems (NHS). Efficient, consolidated EHR schemes operating globally are still a thing of the future; a migratory patient may not have his or her national EHR available in distant territories. This segment of the study focused on this need, and articulated herein is a unified, sound, and secure methodology for achieving much-desired International Interoperability and inclusive efficiency in global EHR implementations, optimising the derived merits of this prime technology. The base model used herein is Health Level 7's (HL7) Electronic Health Record Functional Model (EHR-FM), amongst other similar systems. This solution overarches, and can thus be extrapolated to any ubiquitous EHR environment.

If $\mathrm{F}^{\mathrm{LP}}$ is the EHR-S FM R2 source functional list profile representation and $U$ be the UDA target solution representation. Let $\mathrm{T}^{\mathrm{EHR}}$ be the strict mapping transformation from $\mathrm{F}^{\mathrm{LP}}$ to $\mathrm{U}$ preserving completeness, accuracy, and integrity of the functional list profile information. Then

$$
\begin{gathered}
\mathbf{F}=\sum \mathbf{F}_{1}^{\mathrm{LP}}+\sum \mathbf{F}_{\mathbf{1}}^{\mathrm{LP}}+\sum_{2} \mathbf{F}^{\mathrm{LP}} \mathbf{3}_{\mathbf{3}}+\sum \mathbf{F}^{\mathrm{LP}}{ }_{\mathbf{4}}+ \\
\ldots \ldots \ldots \ldots \ldots \ldots+\mathbf{F}_{\mathbf{k}}^{\mathrm{LP}}
\end{gathered}
$$


It was proved in this study that the mapping $\mathbf{T}^{\mathbf{E H R}} \quad \mathbf{F} \rightarrow \mathbf{U}$ denotes a Complete Transformation, meaning the result of the transformation $\mathrm{T}^{\mathrm{EHR}}$ is a necessary and sufficient target set $\mathrm{U}$ in relation to the source set F. The current EHR approach for modelling electronic health information efficiently supercedes conventional silo-based functional systems, sanctioning its present use in the ITdriven healthcare sector. But, it is empirically proved herein that the enhanced UDA-based EHR model proposed and presented exhibits a significant $100 \%$ improvement and enhancement over its extolled regular EHR counterpart, categorically endorsing its future induction into the IT-based healthcare industry.

HL7's applicability to Sri Lankan Context was studied in depth (Sabar et al., 2015-5). Valued data from over 1600 state medical institutions, ranging from the large city hospitals to the smaller local clinics, were analysed. Prevalent IT technology in these institutions, associated issues and shortcomings, and the desired ITdriven service levels in local healthcare, with emphasis on inter-enterprise interoperability were studied. The envisaged interoperability would levitate healthcare levels, and afford immense benefits all stakeholders, ie., patients, participating healthcare institutions, healthcare personnel, and indeed the healthcare industry itself. The applicability of HL7 in this backdrop, to elevate healthcare services in Sri Lanka, both vertically down the different healthcare-serviceprovider tiers, and laterally in terms of increasing demographic spread, and the allied improvement in the relevant system performance indicators were examined. The proposed Computerette solution, which is an economical, low-cost, self-contained, minimal hardware configuration is an excellent alternative to full-blown computer environments; it provides cost-effective accessibility to HL7 implementation deriving and capitalizing on the immense benefits accruable, at bare minimum overall cost. It is an excellent choice especially for the more backward economies. In the local context, it was determined that $88.89 \%$ of the Ministry of Health's $(\mathrm{MOH})$ concerns with respect to its eHealth mission and its implementation are efficaciously and consummately fulfilled by the proposed HL7-based solution, a significantly high "always go" percentage and a convincing reason for adopting the HL7-SAIF standard nationwide.

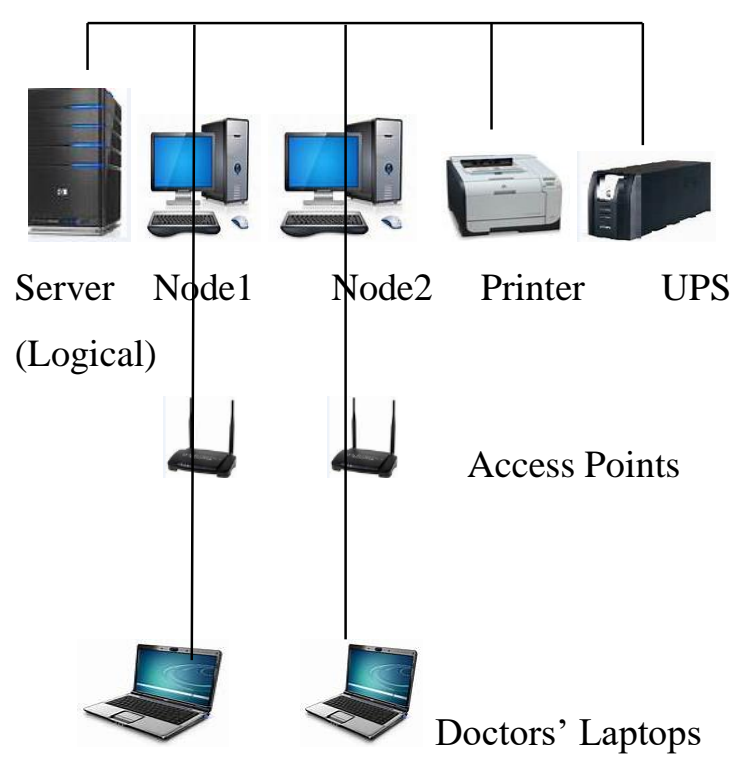

Figure 4. A Model Computerette with Two Client Nodes (Sabar et al., 2015-5)

The study employed timely and relevant data collected about the healthcare sector in Sri Lanka, but the findings are ubiquitous and overarching, seamlessly extrapolatable to the Asian and greater global contexts as well.

\section{CONCLUSION}

The preceding sections articulated HL7_SAIF "case technology and research" highlighting its merits and demerits in design and application. HL7 for example requires a few design improvements heading towards optimization. As recommended in (Landgrebe et al., 2011), HL7 should adopt a tested upper ontology 
framework and an efficient, scientifically wellfounded, modular, and domain-specific modelling language" to surmount this issue.

This research focused primarily on the three principle aspects of IT-based, HL7-oriented healthcare systems development. Enhancement in the specifications generation subprocesses would obviously pervade, promoting highercalibre specifications generation, and subsequent system development. Improvements in implementation with innovation towards globally-consolidated Electronic Health Records (EHRs) would exponentially enhance the quality, availability, and delivery of precision healthcare information to participating stakeholders. Finally, the abounding merits generated, would aggregate synergistically to create complete, efficient, but cost-effective solutions promoting the standard's proliferation, and broadbasing its acceptance and implementation, locally and globally.

SAIF also had concerns in design like in the Specification Stack of its Enterprise Conformance and Compliance Framework (ECCF). However, this segment of research focused purely on HL7, its applicability to the local Sri Lankan context, and issues associated with its specifications development process and subsequent implementation.

It is believed that these refactored enhancements would permeate to the greater HL7-SAIF technology and its applications. Obviously, any definitive and comprehensive solution requires fixes to be applied to both technologies at the points of error detection and would constitute a separate, consummate, research effort.

The remediation effort articulated herein however would go a long way in making HL7SAIF the ubiquitous standard of choice in healthcare-related electronic information exchange.

\section{REFERENCES}

HILERA JR \& RUIZ F. Ontologies in Ubiquitous Computing, International Conference on Ubiquitous Computing, Spain, June 2006.

HL7 (2010) HL7 Services-Aware Interoperability Framework (SAIF), Health Level 7, April 2010.

HL7 (2011) HL7 News, HL7, May 2011.

HL7

(2012)

http://www.diatomenterprises.com/technologies /health-level-7.aspx

HL7

http://en.wikipedia.org/wiki/HL7_Services_Aw are_Interoperability_Framework

HL7 (2014) http://hl7-watch.blogspot.com/

HL7-SAIF (2011) Architectural Review Work Group, HL7 Service-Aware Interoperability Framework - Canonical Definition, Release 1, HL7 Informative Document, September 2011.

LANDGREBE J \& SMITH B. http://ontology.buffalo.edu/smith/articles/HL7-

SAIF.pdf. 2011.

MEAD, CHARLES N. The Service-Aware Interoperability Framework (SAIF) : Making Cross-Boundary Interoperability a First Class Citizen, Service Technology Magazine, January 2012.

NCI

https://wiki.nci.nih.gov/display/VCDE/Introduct ion+to+SAIF+and+ECCF

NYKANEN P. Ontologies in eHealth, Department of Computer Sciences, University of Tampere, February 2010.

SABAR MI, JAYAWEERA PM \& EDIRISURIYA EATA. Requirements Traceability Interpolation for HL7 
Specifications Generation, PGIS ResCon 2015, University of Peradeniya, Sri Lanka, Oct 2015.

SABAR MI, JAYAWEERA PM \& EDIRISURIYA EATA. "Techno-Platform Independence Interpolation in HL7 Ontologies", eHealth Asia 2015, Colombo, Sri Lanka, Oct 2015.

SABAR MI, JAYAWEERA PM \& EDIRISURIYA EATA. "International Interoperability Through Unified Universal HL7 v3 Green Messaging", International Conference on Advances in ICT for Emerging Regions - ICTer 2015, Colombo, Sri Lanka, Aug 2015.

SABAR MI, JAYAWEERA PM \& EDIRISURIYA EATA. Interpolated International Interoperability and Inclusive Efficiency in Ubiquitous Electronic Health Records (EHRs), Asian Transactions on Computers journal, Vol. 05, Issue 04, Aug 2015.

SABAR MI, JAYAWEERA PM \& EDIRISURIYA EATA. Overarching HL7 Interpolation for Asian Interoperability, Asian Transactions on Computers journal, Vol. 05, Issue 03, Jun 2015. 